\title{
Categorisation of Panic Disorder by Time-Frequency Methods
}

\author{
Hubert Dietl ${ }^{1}$, Stephan Weiss ${ }^{1}$ \\ ${ }^{1}$ Dept. Electronics \& Computer Science, University of Southampton, UK \\ hwd, s.weiss@ecs.soton.ac.uk
}

\begin{abstract}
Anxiety patients that are presented with neutral and panic disorder triggering stimuli show different event-related brain potentials (ERP) within the electroencephalogram (EEG). In this paper, we investigate this difference by time-frequency (TF) revealing transforms leading to an identification of a small number of significant parameterising coefficients to be able to differentiate between the presented stimulus categories.
\end{abstract}

\section{INTRODUCTION}

Individuals with panic disorder are characterised by an abnormal fear of certain anxiety connected sensations such as palpitation, breathlessness, or dizziness [1]. The research into this disorder has led to studies investigating its symptoms by means of appropriate stimulation and measurement of the subsequent ERP $[2,3]$. In this context, visual stimulation has been performed with words causing panic disorder, whereby the EEG can be recorded showing event related potentials. Previous studies have resulted in revealing a low frequent transient waveform appearing approximately $300 \mathrm{~ms}$ after stimulus onset as a distinctive characteristic which is referred to as P300.

Analysis of variances (ANOVA) [4] is one method of detecting the P300 in panic disorder and normal response ERP. Since the P300 has a transient behaviour, the application of time frequency (TF) analysis appears well suited, as it takes both spectral and temporal information into account [5]. In this paper we aim to investigate various transforms - such as wavelet, wavelet packet, and Gabor transforms - with respect to their suitability for revealing the TF characteristics of the transient P300. We further optimise these transforms such that the distinction between panic disorder and normal responses is concentrated in only few transform coefficients, to which a statistical test can be applied.

The paper is organised as follows. Sec. 2 will introduce the background and experimental conditions under which panic disorder data was obtained. In Sec. 3 suitable TF transforms will be reviewed, which can pa- rameterise the elicited event related potentials, while Sec. 4 discusses a method to isolate indicative parameters, which can be used for distinguishing between panic disorder and normal EEG. Finally, test results and conclusions are presented in Secs. 5 and 6 .

\section{PANIC DISORDER ERP}

The panic disorder ERP were measured for an anxiety patient who was presented with fear-inducing or neutral words tachistoscopically at the perception threshold of panic disorder. The patient's perception threshold for correctly identifying $50 \%$ of the words was determined with neutral words not used in the experiment. It can be assumed that the patient will recognise a greater number of anxiety words given at his perception threshold than neutral words [4]. Thus, it can be expected that the EEG exhibit an difference when neutral and anxiety words are presented.

The EEG was measured at the vertex electrode $(\mathrm{Cz})$ synchronously to the stimuli, whereby the recordings were started $100 \mathrm{~ms}$ before the onset of the visual word stimulus. The data exemplary analysed in this study contains 24 neutral word presentations and 24 anxiety word presentations to one panic patient. Fig. 1 shows the average over the stimulus-synchronous EEG in reaction to the 24 words presented for each word category. The figure reveals a difference in the two av-

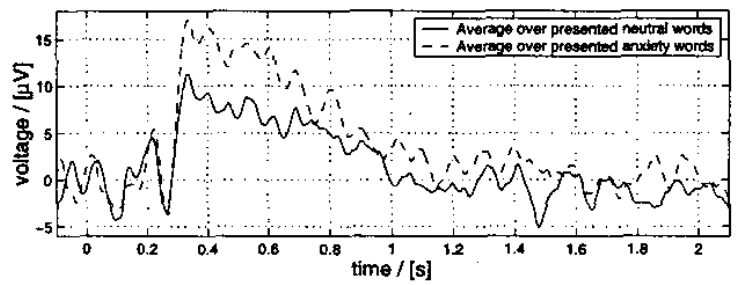

Fig. 1. Average over 24 EEG segments showing responses to anxiety related and neutral stimuli at the perception threshold. 

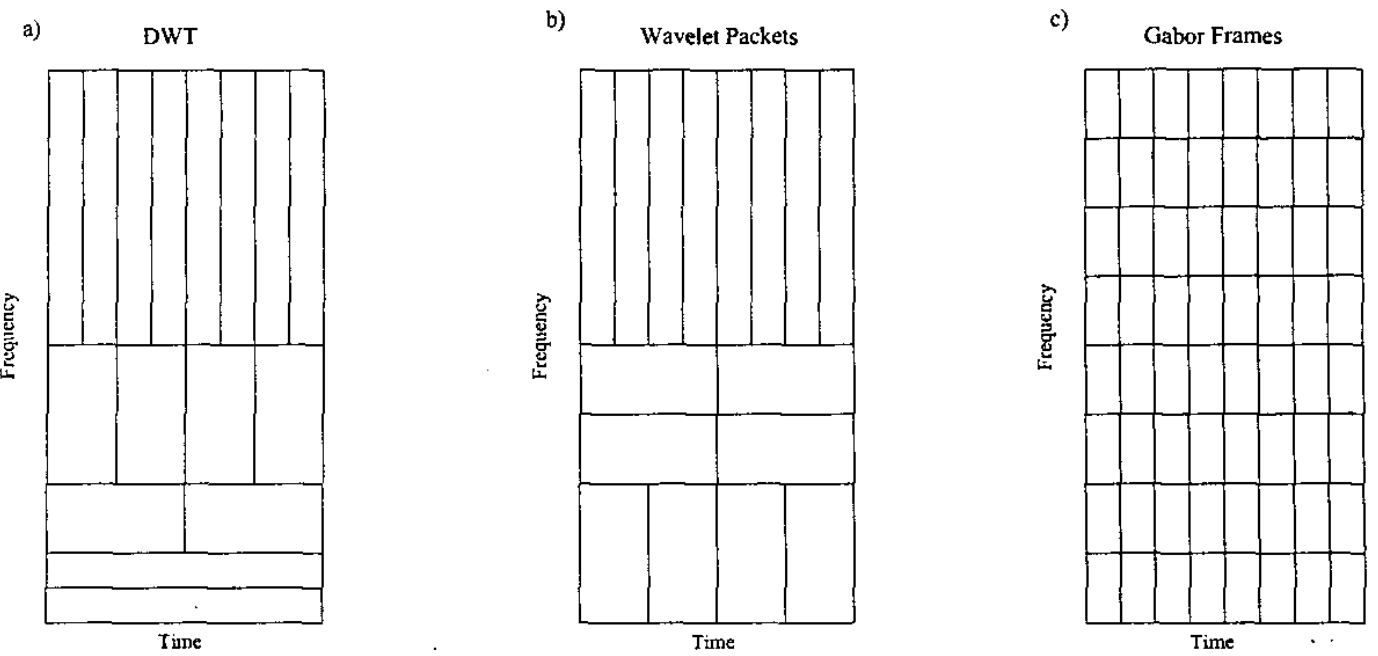

Fig. 2. Time-frequency tiling by a) DWT, b) a sample WP decomposition, and c) a GF decomposition.

erages with a stronger P300 and more positive EEG until approximately $t=700 \mathrm{~ms}$ in the panic disorder related data.

\section{PARAMETERISING TRANSFORMS}

To take the transient nature of the ERP waveforms in Fig. 1 into account, TF transforms are used for parameterisation of the data. To capture the impulsive rise of the P300, TF transforms with a good time resolution are required. The discrete wavelet transform (DWT) however generally yields a good frequency resolution and poor time resolution at low frequencies, resulting in a too coarse time segmentation in the frequency range of interest. Therefore, we concentrate on wavelet packet (WP) transform, whose level of decomposition can be adapted to fit the nature of the data, as well as the Gabor Frame (GF) decomposition, which yields a uniform tiling of the TF plane and hence can provide a desired resolution in a specific TF segment. Fig. 2 show's an example for the TF characteristics of the DWT, the WP and the GF.

The WP is based on Mallat's wavelet [5], whereby the decomposition level of the transformation is adapted to minimise the entropy of the average ERP curves in Fig. 1. The GF decomposition is based on an oversampled filter bank with a flexible number of channels constructed according to [6], whereby the channel number is selected in order to minimise the transform coefficients' entropy when applied to the average ERP curves. Both transformations are operating on finite length EEG segments and are implemented with symmetric boundary extensions $[7,8]$.
The application of the WP and GF transforms leads to a parameterisation of the ERP data whereby the features of the ERP are expressed in as few coefficients as possible. Within these ERP-parameterising coefficients, those that represent a significant difference between the two data sets need to be identified next.

\section{DIFFERENCE EVALUATION}

Based on the parameterisations introduced in the previous section, we want to identify coefficients that allow us to differentiate between the presented anxiety related and neutral words in this section.

\section{$4.1 F$-test}

Prior to the selection of significant coefficients that represent the main characteristics of the data, an $F$ test [9] is conducted to determine which method is used to identify them. The aim of this test is to determine whether two data sets are sampled from normal distributions with the same variances. If a value for the significance level $P$ of lower than 0.05 is obtained by the $F$-test, we conclude that the hypothesis is rejected and the two data sets are sampled from normal distributions having different variances. The value of $P=0.05$ is a limit commonly used in medical research [9]. Comparing sets $\mathbf{x}_{p}$ and $\mathbf{x}_{n}$ containing the panic disorder and neutral ERP response coefficients for one specific transform coefficient across all 24 measurements, the $F$-value is given by [9]

$$
F=\frac{\sigma_{p}^{2}}{\sigma_{n}^{2}},
$$


with $\sigma_{p}^{2}$ and $\sigma_{n}^{2}$ being the variances of the two data sets. To receive the significance level $P$ for the $F$-test, we need to define the degrees of freedom for the two data sets according to

$$
\begin{aligned}
& \nu_{p}=N_{p}-1 \text { and } \\
& \nu_{n}=N_{n}-1
\end{aligned}
$$

with $N_{p}=N_{n}=24$ being the number of samples, $\nu_{p}$ the degrees of freedom for the panic data set and $\nu_{n}$ the degrees of freedom for the neutral data set. With the $F$ value defined by (1) and the degrees of freedom $\nu_{p}$ and $\nu_{n}$, the significance level $P$ for the $F$-test can be determined from lookup tables in literature, e.g. [9]. If the outcome of the $F$-test confirms that the two data sets are sampled from distributions with equal variances, we can subsequently conduct a $t$-test to determine distinctive coefficients. If the result of the $F$-test is that the underlying distributions from which the two data groups are sampled possess different variances we conduct a $u t$-test. The $t$-test and the $u t$-test are defined in the next subsection.

\section{2 $T$ - and $U T$-Tests}

The $t$-test gives the probability that two data sets sampled from potentially two different distributions with identical variance possess different mean values, for which a significance is returned. The $t$-value is defined as [10]

$$
t=\frac{\overline{\mathbf{x}}_{p}-\overline{\mathbf{x}}_{n}}{\sqrt{\frac{\sigma_{p}^{2}}{N_{p}}+\frac{\sigma_{n}^{2}}{N_{n}}}}=\frac{\overline{\mathbf{x}}_{p}-\overline{\mathbf{x}}_{n}}{\sigma \sqrt{\frac{1}{N_{p}}+\frac{1}{N_{n}}}},
$$

with $\sigma^{2}=\sigma_{p}^{2}=\sigma_{n}^{2}$. The values $\overline{\mathbf{x}}_{p}$ and $\overline{\mathbf{x}}_{n}$ represent the means for the two data sets, according to

$$
\overline{\mathbf{x}}_{i}=\frac{1}{N_{i}} \cdot \sum_{n=0}^{N_{i}-1} \mathbf{x}_{i}[n], \quad i \in\{p, n\} .
$$

The $t$-value also corresponds to a certain significance level $P$, which can be looked up from tables [10], with the degrees of freedom defined by $\nu_{t}=\nu_{p}+\nu_{n}=$ $N_{p}+N_{n}-2$. A smaller value for $P$ indicates that the data sets have a significantly different mean. For example, for $P=0.01$ the probability that the differences in the means are due to a sampling error is $1 \%$. For our study, a significance level of $P=0.01$ was used to identify distinctive coefficients. The two tested distributions were the distributions for a specific transform parameter over the presented 24 neutral and anxiety words, respectively.

For the case that the $F$-test yields a difference in variances such that the $t$-test cannot be used, we apply a $u t$-test for unequal variances defined as

$$
u t=\frac{\overline{\mathbf{x}}_{p}-\overline{\mathbf{x}}_{n}}{\sqrt{\frac{\sigma_{p}^{2}}{N_{p}}+\frac{\sigma_{n}^{2}}{N_{n}}}} .
$$

According to [11], for data sets sampled from distributions with unequal variances, the $t$ distribution can be approximated by the $u t$ value if the $t$ table is entered at the following defined degree of freedom:

$$
\nu_{u t}=\frac{\left(\sigma_{p}^{2} / N_{p}+\sigma_{n}^{2} / N_{n}\right)^{2}}{\frac{\left(\sigma_{p}^{2} / N_{p}\right)^{2}}{N_{p}-1}+\frac{\left(\sigma_{n}^{2} / N_{n}\right)^{2}}{N_{n}-1}} .
$$

Again, for our study, a significance level of $P=0.01$ was used for the ut-test to identify distinctive coefficients. This test tends to be less powerful than the usual $t$-test, since it uses fewer assumptions [9]. As it will be shown in the application in the next section, all identified distinctive coefficients there have been isolated by the $t$-test. The main purpose of the $u t$-test is to have an analysis tool for all coefficients at hand whether they show equal variances or not.

To confirm the results obtained by $t$-tests or $u t$-tests, a back test can be performed based on an ROC analysis as shown in the next subsection.

\subsection{ROC Analysis Back Test}

According to [12], a good measure for differentiation between two distributions are ROC curves, since the area under the ROC "curve measures the separability independent of the selection of any threshold.

Here, we make use of it to back test the results obtained by $t$-tests or $u t$-tests. The back test is performed as follows. For every coefficient received, the area under the ROC curve is measured. For this measure, two Gaussian distributions are generated. From these distributions, the same number of random samples as in the preceding $t$-test or $u t$-test are taken out and based on a $t$-test or $u t$-test, the significance level is calculated for these samples originating from the Gaussian distributions. This calculation is repeated with random samples from the distributions and the significance level is averaged until it converges. Tab. 1 shows some areas under the ROC curve and their corresponding significance levels $P$.

In most social research a significance level of $P=$ 0.05 is used to determine differences between two sets of data. Therefore, if in our study the area under ROC curve is equal or greater than 0.72 , we will conclude that this coefficient has passed the back test and separates our data adequately enough. 


\begin{tabular}{|c|c|}
\hline Area under the ROC curve & Significance level $P$ \\
\hline 0.74 & 0.028 \\
0.72 & 0.046 \\
0.70 & 0.067 \\
\hline
\end{tabular}

Tab. 1. Area under ROC curve and significance levels $P$.

\section{TEST AND DISCUSSION}

As discussed in Sec. 3 and Sec. 4, we have different transform methods and a procedure to identify significant coefficients to being able to separate between presented neutral and anxiety words. In the following, we will discuss the used transforms and present the results for separability which we obtained for the data described in Sec. 2 .

\subsection{Transform Adjustment}

The optimal decomposition structure for the WP is found over minimising the entropy as described in Sec. 3. The decomposition depth was limited to have at least 16 coefficients in one decomposition level as further decomposition would lead to a too coarse time segmentation. In terms of the Gabor transform, various filters were tested and it was found that using a prototype filter with length of 224 , a frequency segmentation of 32 uniform scales and a time segmentation of 14 for the oversampling shows the best results. The resulting approximate distribution of the coefficient energies in the TF plane is risualised in Fig. 3.
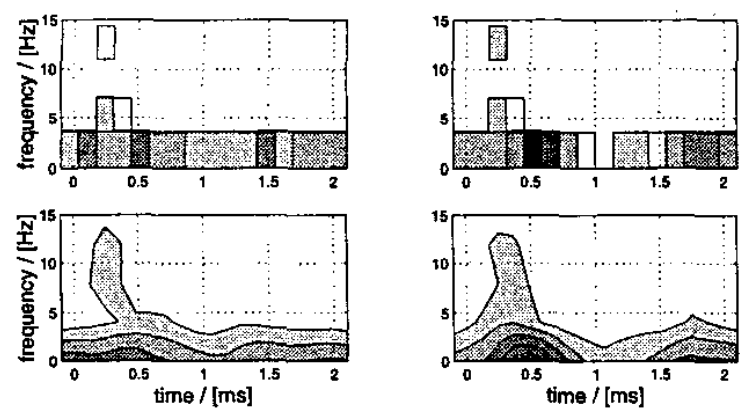

Fig. 3. Average coefficient energy for (left) neutral words and (right) panic order related words using (top) WP and (bottom) Gabor transforms.

\subsection{Identified Coefficients and Difference Com- parison}

The coefficients to which the difference evaluation is applied were preselected whereby only coefficients are considered which contain $85 \%$ of the total energy. This is reasonable, as it reduces the probability to identify coefficients that contain noise only. The value of $85 \%$ results from not considering coefficients that are located above $15 \mathrm{~Hz}$ in the TF plane, see Fig. 3.

Fig. 4 shows the resulting coefficients when performing the difference evaluation on the parameterised data. We see that two coefficients (black and grey) for both
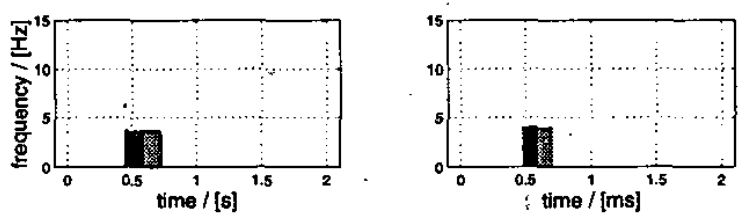

Fig. 4. Resulting coefficients for (left) WP and (right) Gabor transforms.

transforms are identified. They cover approximately the area of the P300 slow wave as it is expected in Sec. 2. They are all identified via a $t$-test according to a prior $F$-test whereby the threshold for the significance level for the $F$-test was $P=0.05$, and for the $t$-test, it was set to $P=0.01$ as mentioned in the previous section.

Fig. 5 shows the difference of the averages of the neutral and anxiety EEG compared with its parameterisation by the identified coefficients for the two investigated transforms. It can be observed that the two identified coefficients parameterise the P300 area very
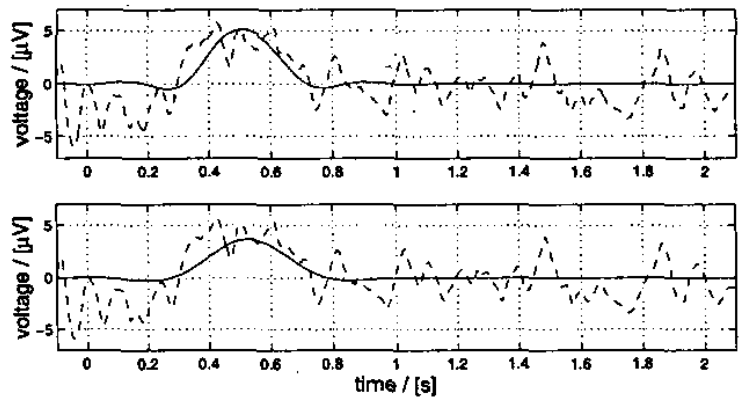

Fig. 5. Difference of neutral and anxiety EEG data compared with its parameterisation by the two identified coefficients for (top) WP and (bottom) Gabor transforms. 
well for both transforms.

To show the results of the back test for the identified coefficients, Tab. 2 indicates the ROC curve analysis for these coefficients. Comparison with Tab. 1 yields

\begin{tabular}{|c||c|c|}
\hline \multicolumn{1}{|c||}{ Coefficient } & \multicolumn{2}{c|}{ Transform } \\
WP & GF \\
\hline black & 0.73 & 0.73 \\
grey & 0.72 & 0.72 \\
\hline
\end{tabular}

Tab. 2. Area under ROC curve for the identified coefficients.

that all coefficients obtained show an equal or greater value than 0.72 and therefore, pass the back test what justifies the use of the respective transforms. Recapitulating, it can be said that with both transforms an adequate separation of data of both categories, namely presented neutral and anxiety words, can be achieved. These results were contrasted to a difference evaluation applied to the time domain and frequency domain data, where the latter is calculated via a discrete Fourier transform, for which only poor separability was achieved.

\section{CONCLUSIONS}

We have presented a WP and Gabor transforms analysis comparison for parameterising ERP with the aim of differentiating between presented neutral and anxiety words to a patient with panic disorder. We have motivated the use of TF methods, and proposed an approach to obtain distinctive transform coeffcients, whereby the results were verified by different tests for different cases. It was shown that the presented TF transforms can be used with good results to classify panic disorder via analysis of ERP.

\section{REFERENCES}

[1] D. M. Clark, "A cognitive approach to panic," Behaviour Research and Therapy, vol. 24, pp. 461$470,1986$.

[2] E. A. Kostandov and Y. L. Arzumanov, "Average cortical evoked potentials to recognised and nonrecognised verbal stimuli," Acta Neurobiologica Experimentalis, vol. 37, pp. 311-324, 1977.

[3] E. Naumann, D. Bartussek, O. Diedrich, and M.E. Laufer, "Assessing cognitive and affective information processing functions of the brain by means of the late positive complex of the event-related potential," Journal of Psychophysiology, vol. 6, pp. 285-298, 1992.

[4] P. Pauli, G. Dengler, G. Wiedemann, P. Montoya, H. Flor, N. Birbaumer, and G. Buchkremer, "Behavioral and Neurophysiological Evidence for Altered Processing of Anxiety-Related Words in Panic Disorder," Journal of Abnormal Psychology, vol. 106, no. 2, pp. 213-220, 1997.

[5] Stephane G. Mallat, "Multiresolution Approximations and Wavelet Orthonormal Bases of $L^{2}(R)$," Transactions of the American Mathematical Society, vol. 315, no. 1, pp. 69-87, September 1989.

[6] M. Harteneck, S. Weiss, and R. W. Stewart, "Design of Near Perfect Reconstruction Oversampled Filter Banks for Subband Adaptive Filters," IEEE Transactions on Circuits \& Systems II, rol. 46, no. 8, pp. 1081-1086, August 1999.

[7] G. Strang and T. Nguyen, Wavelets and Filter Banks, Wellesley-Cambridge Press, Wellesley, MA, 1996.

[8] H. Dietl, S. Weiss, and U. Hoppe, "Comparison of Transformation Methods to Determine Frequency Specific Cochlear Hearing Loss Based on TEOAE," in Digest 02/110 IEE Colloquium on Medical Applications of Signal Processing, IEE, London, October 2002, pp. 16/1-16/6.

[9] P. Armitage, G. Berry, and J.N.S. Matthews, Statistical Methods in Medical Research, Blackwell Science, Oxford, fourth edition, 2002.

[10] I.N Bronstein and K.A. Semendjajew, Taschenbuch der Mathematik, Verlag Harri Deutsch, Thun und Frankfurt/Main, 23rd edition, 1987.

[11] F.E. Satterthwaite, "An approximate distribution of estimates of variance components," Biometrics Bull, vol. 2, pp. 110-114, 1946.

[12] J. A. Hanley and B. J. McNeil, "The Meaning and Use of the Area under a Receiver Operating Characteristic (ROC) Curve," Radiology, vol. 143, pp. 26-36, 1982. 